\title{
Nerve Ultrasound as a Decisive Tool in Nonsystemic Vasculitic Neuropathy: A Case Report
}

\author{
Daniela Leupold ${ }^{a} \quad$ Ansgar Felbecker $^{a} \quad$ Barbara Tettenborn $^{a}$ \\ Thomas Hundsberger ${ }^{a, b}$ \\ Departments of ${ }^{a}$ Neurology and ${ }^{b}$ Haematology and Oncology, Cantonal Hospital, \\ St. Gallen, Switzerland
}

\section{Key Words}

Nerve ultrasound · Nonsystemic vasculitic neuropathy · Systemic vasculitic neuropathy

\begin{abstract}
Introduction: The additional value of peripheral nerve ultrasound in acquired immunemediated neuropathies has recently been reported. Its impact in vasculitic neuropathy is yet to be defined. We report electrophysiological and nerve ultrasound studies in a patient with nonsystemic vasculitic neuropathy at first diagnosis and in response to immunosuppression. Case Report: A 44-year-old female presented with painful neuropathy and weakness of the intrinsic hand muscles. Electrodiagnostic studies revealed severe axonal neuropathy of the nerves of the left arm. On nerve ultrasound, massive and patchy swelling of these nerves was detected. Clinical, laboratory, and radiological evidence of nonneuromuscular involvement and systemic vasculitic diseases was absent. Hence, nonsystemic vasculitic neuropathy was diagnosed without the possibility of histological verification. After 6 months of systemic immunosuppression with steroids and cyclophosphamide, clinical symptoms improved in parallel with neurosonography. In contrast, electrophysiological studies remained pathological despite clinical improvement. Conclusions: Neurosonography studies in nonsystemic vasculitic neuropathy are rare but might be an ancillary technique to guide noninvasive diagnosis and therapeutic monitoring. Morphological analysis of nerves and changes in response to treatment could be well visualized. Additionally, neurosonography might be useful to target nerve biopsy.


Leupold et al.: Nerve Ultrasound as a Decisive Tool in Nonsystemic Vasculitic Neuropathy: A Case Report

\section{Introduction}

Vasculitic neuropathies are a heterogeneous and rare group of peripheral nerve disorders. They are categorized into two groups: primary nonsystemic vasculitic neuropathies (NSVNs) affect only the peripheral nervous system, whereas secondary vasculitic neuropathies evolve in the context of a primary systemic vasculitis. NSVNs affect the minority of cases, accounting for 15\% [1]. Classification criteria are not standardized, but recently, comprehensive diagnostic criteria have been proposed [2]: (1) clinical evidence of neuropathy; (2) electrodiagnostic changes consistent with axonal neuropathy; (3) nerve or nerve/muscle biopsy diagnostic of vasculitis; (4) without clinical, laboratory, or radiological evidence of nonneuromuscular involvement; (5) no identified other disease etiology, and (6) no systemic disease predisposing to vasculitis. As with systemic vasculitic neuropathy (SVN), NSVN most commonly presents as mononeuritis multiplex. Nevertheless, it can also occur as symmetrical polyneuropathy [3] and asymmetrical sensory or motor neuropathy [4]. Thus, NSVN should be suspected particularly if an axonal neuropathy is asymmetric, multifocal, progressive, and painful and is associated with a paresis. The peroneal, tibial, and ulnar nerves are mostly affected. Almost all vasculitic neuropathies develop acutely or subacutely; a chronic development over years is quite rare. Unspecific symptoms, such as weight loss, fever, or fatigue, have been reported in $80 \%$ of cases in systemic vasculitis and to a lower percentage also in NSVNs [4].

NSVN is a challenging diagnosis. An extensive workup [i.e. clinical examination, nerve conduction studies (NCS), electromyography, and laboratory tests including urine analysis] is necessary mainly to exclude different etiologies. A definitive diagnosis can only be achieved by a nerve biopsy. However, it is limited by a low sensitivity. Sural nerve biopsy was diagnostic in $37-58 \%$ of patients with SVNs [5, 6] but only in $25 \%$ of those with NSVNs [5]. Moreover, sensorimotor and pure motor nerves cannot be biopsied without the risk of additional neurological disabilities. Therefore, alternative and noninvasive methods to determine the diagnosis are much appreciated. The value of ultrasound of the peripheral nerves in acquired immune-mediated neuropathies has been reported in detail [7]. The impact of neurosonography in vasculitic neuropathy has just recently been described. Grimm et al. [8] could demonstrate morphological changes in vasculitic neuropathy with focal nerve enlargement but no pronounced general nerve enlargement as expected in immune-mediated demyelinating neuropathies (e.g. chronic inflammatory demyelinating neuropathy). Our case report highlights neurosonography changes in a patient with NSVN at first diagnosis and in response to immunosuppression.

\section{Methods}

Neurosonography was performed using a Philips EPIQ 5 imaging system with an 18$\mathrm{MHz}$ linear-array transducer. All peripheral nerves were measured bilaterally (median, ulnar, radial, brachial plexus, tibial, fibular, and sural nerves). Each nerve was scanned according to standard protocols, and the maximal cross-sectional area (CSA) was measured at the inner border of the hyperechoic epineural rim. Published normal values adapted to local standards were used for comparison [9].

NCS were performed bilaterally with modern electrodiagnostic equipment (Dantec ${ }^{\mathrm{TM}}$ Keypoint ${ }^{\circledR}$ ) on the same day as the sonographic evaluation. Normal values were derived from institutional standards. Motor nerve conduction velocities (NCVs) of the median, ulnar, 
Leupold et al.: Nerve Ultrasound as a Decisive Tool in Nonsystemic Vasculitic Neuropathy: A Case Report

radial, peroneal, and tibial nerves were investigated. Sensory NCVs were determined for the median, ulnar, radial, peroneal, and sural nerves.

\section{Case Description}

A 44-year-old female presented with right-sided severe neuropathic pain at the scapula radiating along the dermatomes $\mathrm{C8} / \mathrm{Th} 1$ as well as burning pain at the fingertips, which gradually developed over 1 month. Additionally, the patient complained of weakness of the right hand. Two weeks before admission, she had noticed painful dysesthesias in the left fingers and the right knee (cutaneous branches of the femoral nerve).

The clinical examination revealed severe paresis of the right intrinsic hand muscles (Medical Research Council grade 3 paresis) and sensory deficits in the territory of both the median and right axillary nerves. There was a pronounced allodynia along the course of the upper arm nerves. Fever, night sweats, and weight loss were not reported. The serum and urine laboratory tests and cerebrospinal fluid analysis were unremarkable without evidence of an infectious, inflammatory, or systemic vasculitic disease. The magnetic resonance images of the brain, the cervical spine, and plexus brachialis with and without gadolinium were also normal. NCS revealed a severe axonal neuropathy of the right median and ulnar nerve with reduced compound muscle action potential amplitudes and sensory nerve action potentials, while NCVs were in the normal range. Electromyography of the paretic muscles (e.g. first dorsal interosseous and abductor pollicis brevis) showed a neurogenic pattern including spontaneous muscle fiber activity and polyphasic, extended, and high-amplitude motor unit action potentials, whereas clinically unaffected muscles innervated by different nerves were normal. On nerve ultrasound, multifocal swelling of the right median and ulnar nerves with enlarged CSAs was detected. Interestingly, we frequently found isolated enlarged nerve fascicles in the affected nerves. Beside enlargement, the affected nerves showed a decreased echogenicity with a hyperechogenic, thickened epineurium (fig. 1, fig. 2). Focally increased CSA was also detected in the electrophysiologically unaffected left median und ulnar nerves. On the side of nerve enlargement, compression with the ultrasound probe was painful.

In summary, subacute and asymmetric painful neuropathy in different nerve regions, axonal changes at electrodiagnostic examination, and missing clinical, laboratory, and radiological evidence of nonneuromuscular involvement and systemic vasculitic diseases as well as no other identified etiology strongly point to the diagnosis of NSCN [2]. However, a definitive diagnosis could not be achieved, as nerve biopsy was not feasible due to the affected sensorimotor nerves (i.e. median and ulnar nerves). Other terminal sensory nerves suitable for nerve biopsy (i.e. sural or radial nerves) were neither clinically, electrophysiologically nor sonographically affected.

As initial intravenous high-dose steroids were insufficient to alleviate the clinical symptoms, immunosuppression was escalated to a combined treatment with 6 monthly courses of intravenous cyclophosphamide $\left(500 \mathrm{mg} / \mathrm{m}^{2}\right.$ body surface). After 2 months of treatment, neuropathic pain greatly improved, and neurological deficits stabilized. Finally, after tapering of immunosuppression, muscle strength returned to normal. However, NCS remained unchanged, showing persistent and severe axonal damage of the right median and ulnar nerves. In contrast, neurosonography findings improved remarkably. The pathological enlargement of single nerve fascicles diminished, and the overall swelling of the affected nerves was reduced; nerve echogenicity remained decreased in the affected nerves (fig. 3). For long-term immunosuppression, therapy was switched to azathioprine monotherapy ( $3 \mathrm{mg} / \mathrm{kg}$ body weight daily). 
Leupold et al.: Nerve Ultrasound as a Decisive Tool in Nonsystemic Vasculitic Neuropathy: A Case Report

\section{Discussion}

Superficial peripheral nerves can thoroughly be investigated by high-resolution neurosonography. Hence, neurosonography might advance to become a useful complementary tool to electrodiagnostic studies, as it allows the detailed visualization of nerve fascicles and small structural changes [10]. The value of peripheral nerve ultrasound has already been established in acquired immune-mediated demyelinating neuropathies like acute and chronic inflammatory demyelinating neuropathy as well as in inherited demyelinating neuropathies $[7,11]$. Increased CSAs of the affected nerves, especially in the proximal extremities, and patchy nerve enlargement at the site of conduction blocks are the hallmarks of sonographic findings in demyelinating neuropathies. The distribution pattern of nerve alterations (diffuse, generalized, or focal) can give etiological clues of demyelinating neuropathies [12]. In contrast, ultrasound is rarely abnormal in diffuse axonal polyneuropathies. Hence, neurosonography seems to be useful for the morphological delineation between axonal and demyelination neuropathies [13].

In contrast, vasculitic neuropathy may be an exception in the context of these findings. In our case, we illustrated pronounced changes of different nerves using ultrasound. Besides multifocal nerve swelling, we found impressively enlarged nerve fascicles in the affected nerves. The affected nerves showed a decreased echogenicity with a hyperechogenic, thickened epineurium, which persisted even after immunosuppression. Just a few case reports of vasculitic neuropathies are available where focal morphologic lesions were sonographically identified. Ito et al. [14] described abnormalities in tibial nerve vasculitic neuropathy with an enlarged and hypoechoic nerve. Recently, Grimm et al. [8] reported on systematic peripheral nerve measurements in vasculitic neuropathies using ultrasound. In patients with primary or secondary SVN, single fascicle enlargements in clinically affected nerves were detected. Given that nonimmune-mediated axonal neuropathies in general do not show significant nerve enlargement, nerve ultrasound might be a helpful additional tool to NCS in case of asymmetric axonal damage of unknown origin. In comparison with demyelinating neuropathies with generalized nerve enlargements, accentuated in proximal nerve segments, in vasculitic neuropathy mean CSA values are just slightly increased, whereas focal nerve enlargement and particularly fascical swelling are more prominent in these cases. The findings in our case are in agreement with the study by Grimm et al. [8]. The pathophysiology of nerve enlargement and fascicular swelling in vasculitic neuropathies remains unclear. It might be an inflammation of the vasa nervorum, mainly affecting the epineural arteries, leading to small-vessel infarction and thrombosis, focal edema, and consecutive ischemic damage of the axon [15]. The different etiology and rapid time course might explain the peculiar presentation of NSVN compared to other axonal neuropathies.

The main limitation of the differential diagnosis in our case was the missing histological verification of NSVN due to the affected eloquent nerves of the upper extremity. We considered the risk of neurological harm due to a nerve biopsy carefully in view of the low sensitivity of this procedure [6] and decided in favor of a conservative approach. However, asymmetric and painful axonal mononeuropathy multiplex without evidence of an alternative etiology or the presence of clinical and paraclinical parameters of systemic vasculitis raises suspicion of NSVN, as 5 out of the 6 proposed diagnostic criteria were already present [2]. Moreover, by nerve ultrasound we identified morphological findings consistent with the diagnosis of NSVN and documented a sonographic response along with the alleviation of clinical symptoms achieved by intensive systemic immunosuppression. Just recently, different study groups described nerve ultrasound changes in immune-mediated neuropathies after immunoglobulin therapy over time. In chronic inflammatory demyelinating neuropathy 
Leupold et al:: Nerve Ultrasound as a Decisive Tool in Nonsystemic Vasculitic Neuropathy: A Case Report

patients, a correlation between muscle strength and CSA variability could be illustrated [16]; also, in Guillain-Barré syndrome patients, a regression of nerve enlargement might be a good parameter for clinical restitution during follow-up [17]. Therefore, nerve ultrasound might be a promising monitoring method, although literature on proven therapeutic effects of ultrasound in vasculitic neuropathy is missing and sample sizes in immune-mediated neuropathies are small. Further studies need to prove these effects. In summary, nerve ultrasound is a new and noninvasive diagnostic tool for the confirmation of peripheral nerve vasculitis supplementary to electrophysiological studies. It is easy to use in experienced hands, cheap, and probably a better follow-up instrument than NCS or electromyography, as it showed earlier response and a better correlation with the clinical follow-up in our case. Furthermore, nerve enlargement can be detected in clinically and electrophysiologically unaffected nerves.

\section{Statement of Ethics}

The patient gave her informed consent for the publication of this article.

\section{Disclosure Statement}

The authors declare that they have no conflicts of interest.

\section{References}

1 Sampaio L, Silva LG, Terroso G, Nadais G, Mariz E, Ventura F: Vasculitic neuropathy. Acta Reumatol Port 2011;36:102-109.

2 Collins MP, Periquet MI: Non-systemic vasculitic neuropathy. Curr Opin Neurol 2004;17:587-598.

-3 Davies L, Spies JM, Pollard JD, McLeod JG: Vasculitis confined to peripheral nerves. Brain 1996;119:14411448.

4 Collins MP, Periquet MI, Mendell JR, Sahenk Z, Nagaraja HN, Kissel JT: Nonsystemic vasculitic neuropathy: insights from a clinical cohort. Neurology 2003;61:623-630.

5 Dyck PJ, Benstead TJ, Conn DL, Stevens JC, Windebank AJ, Low PA: Nonsystemic vasculitic neuropathy. Brain 1987;110:843-853.

6 Bennett DLH, Groves M, Blake J, et al: The use of nerve and muscle biopsy in the diagnosis of vasculitis: a 5 year retrospective study. J Neurol Neurosurg Psychiatry 2008;79:1376-1381.

7 Kerasnoudis A, Pitarokoili K, Behrendt V, Gold R, Yoon M-S: Nerve ultrasound score in distinguishing chronic from acute inflammatory demyelinating polyneuropathy. Clin Neurophysiol 2014;125:635-641.

8 Grimm A, Décard BF, Bischof A, Axer H: Ultrasound of the peripheral nerves in systemic vasculitic neuropathies. J Neurol Sci 2014;347:44-49.

-9 Zaidman CM, Al-Lozi M, Pestronk A: Peripheral nerve size in normals and patients with polyneuropathy: an ultrasound study. Muscle Nerve 2009;40:960-966.

10 Peer S, Bodner G (eds): High-Resolution Sonography of the Peripheral Nervous System. Berlin/Heidelberg, Springer, 2008.

11 Sugimoto T, Ochi K, Hosomi N, et al: Ultrasonographic nerve enlargement of the median and ulnar nerves and the cervical nerve roots in patients with demyelinating Charcot-Marie-Tooth disease: distinction from patients with chronic inflammatory demyelinating polyneuropathy. J Neurol 2013;260:2580-2587.

$\checkmark 12$ Zaidman CM, Harms MB, Pestronk A: Ultrasound of inherited vs acquired demyelinating polyneuropathies. J Neurol 2013;260:3115-3121.

-13 Grimm A, Heiling B, Schumacher U, Witte OW, Axer H: Ultrasound differentiation of axonal and demyelinating neuropathies. Muscle Nerve 2014;50:976-983.

14 Ito T, Kijima M, Watanabe T, Sakuta M, Nishiyama K: Ultrasonography of the tibial nerve in vasculitic neuropathy. Muscle Nerve 2007;35:379-382.

15 Kararizou E, Davaki P, Karandreas N, Davou R, Vassilopoulos D: Nonsystemic vasculitic neuropathy: a clinicopathological study of 22 cases. J Rheumatol 2005;32:853-858. 
Leupold et al.: Nerve Ultrasound as a Decisive Tool in Nonsystemic Vasculitic Neuropathy: A Case Report

16 Kerasnoudis A, Pitarokoili K, Gold R, Yoon M-S: Nerve ultrasound and electrophysiology for therapy monitoring in chronic inflammatory demyelinating polyneuropathy. J Neuroimaging 2015;25:931-939.

17 Grimm A, Décard BF, Schramm A, et al: Ultrasound and electrophysiologic findings in patients with GuillainBarré syndrome at disease onset and over a period of six months. Clin Neurophysiol 2016;127:1657-1663.
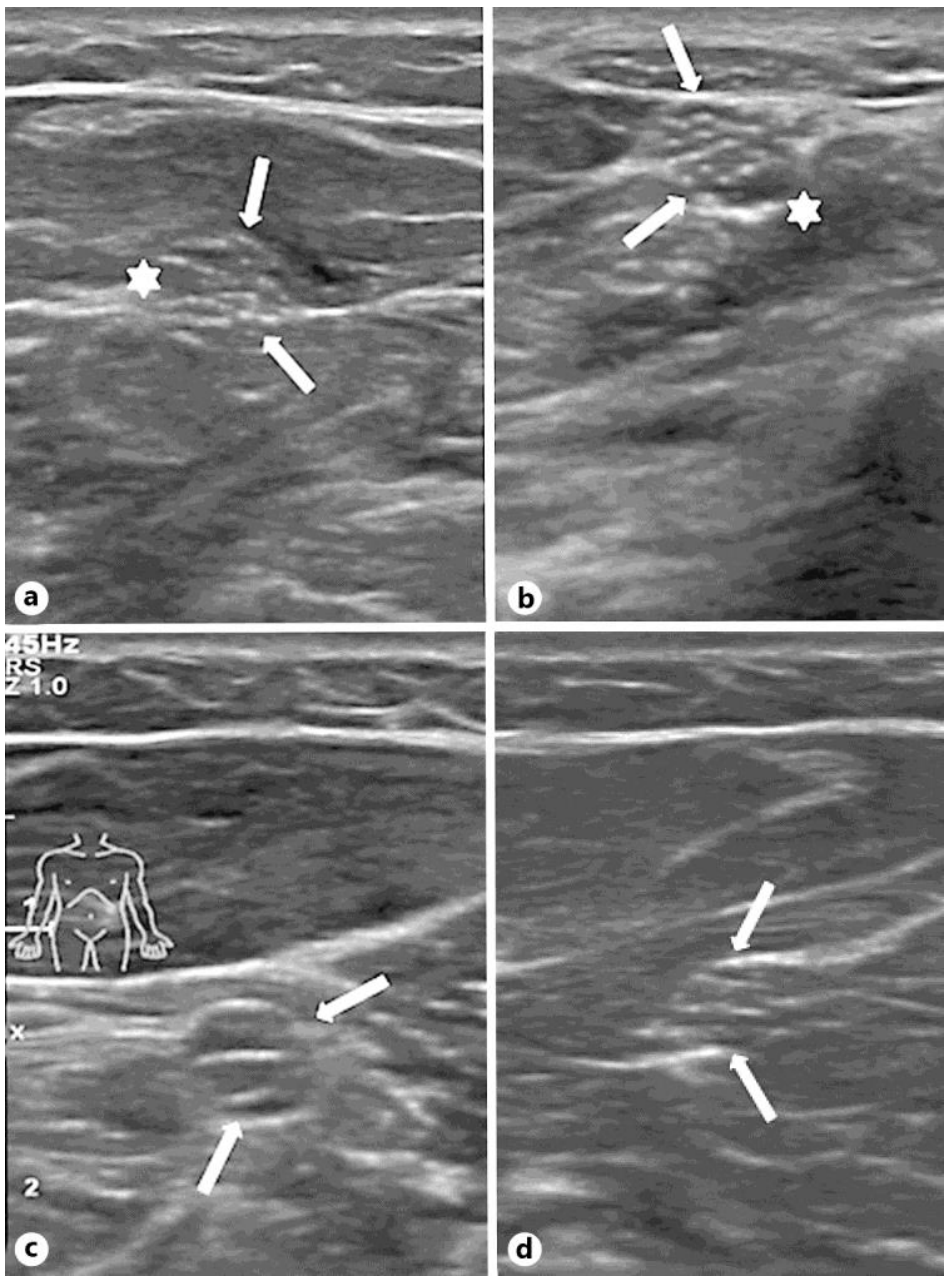

Fig. 1. Axial ultrasound of the right median nerve. a, b Multifocal nerve enlargements at different nerve sites with increased CSA (arrows) and significant interfascicular swelling ( $>2 \mathrm{~mm}^{2}$ ) (asterisks). c Enlarged median nerve (arrows) compared to healthy nerve segment (d) (arrows) on the left side (CSA 12 vs. $8 \mathrm{~mm}^{2}$ ). 


\section{Case Reports in Neurology}

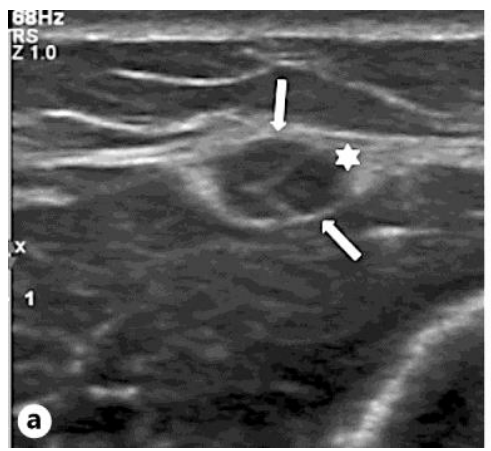

\begin{tabular}{l|l}
\hline Case Rep Neurol 2016:8:108-114 \\
\hline DOI: $10.1159 / 000446314$ & $\begin{array}{l}\text { (c) 2016 The Author(s). Published by S. Karger AG, Basel } \\
\text { www.karger.com/crn }\end{array}$ \\
\hline
\end{tabular}

Leupold et al.: Nerve Ultrasound as a Decisive Tool in Nonsystemic Vasculitic Neuropathy: A Case Report

Fig. 2. Multifocal swelling of the right ulnar nerve (a) with hyperechogenic epineurium (arrow) and interfascicular swelling (asterisks) compared to the healthy ulnar nerve segment on the left in the upper arm (b) (CSA 10 vs. $5 \mathrm{~mm}^{2}$ ).
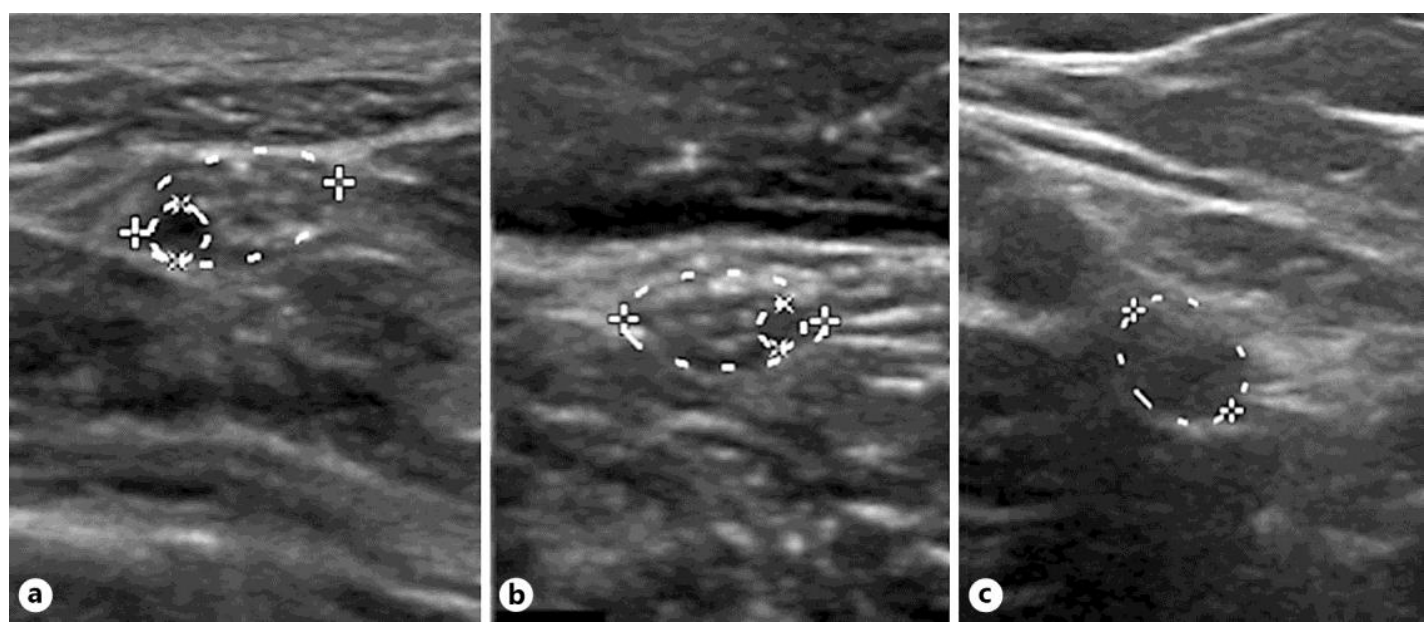

Fig. 3. Axial ultrasound of the median nerve 6 months after treatment with cyclophosphamide. a, b Normal CSA in different nerve segments with persistent mild swelling of single fascicles ( $<1 \mathrm{~mm}$ in diameter). c Normal values of CSA in the upper arm with persistent hypoechogenic nerve structure without discrimination of different fascicles. 